\title{
COMBINATORIAL EFFECT OF D-AMINOACIDS AND TETRACYCLINE AGAINST PSEUDOMONAS AERUGINOSA BIOFILM
}

\author{
H. JAYALEKSHMI, C. HARIKRISHNAN, SAJIN SALI, N. KAUSHIK, NORIN MARY G. VICTUS, R. ANOOP, T. M. \\ SARATH, O. ATHIRA, GEETHA B. KUMAR, BIPIN NAIR*
}

Amrita School of Biotechnology, Amrita Vishwa Vidyapeetham, Amritapuri, Clappana P 0, Kollam, Kerala, India

Email: bipin@am.amrita.edu

Received: 16 Oct 2015 Revised and Accepted: 21 Sep 2016

\begin{abstract}
Objective: The present study attempted to evaluate the anti-biofilm activity of D-amino acids (D-AAs) on Pseudomonas aeruginosa and determine if the combination of D-AAs with tetracycline enhances the anti-biofilm activity in vitro and ex vivo.

Methods: Different D-AAs were tested for antibiofilm activity against wild type $P$. aeruginosa PA01 and two multidrug resistant $P$. aeruginosa clinical strains in the presence of sub inhibitory concentrations of tetracycline using crystal violet microtitre plate assay. Results were further validated using in vitro wound dressing and ex vivo porcine skin models followed by cytotoxicity and hemocompatibility studies.

Results: D-tryptophan ( $5 \mathrm{mmol}$ ) showed $61 \%$ reduction in biofilm formation of P. aeruginosa. Interestingly combinatorial effect of $5 \mathrm{mmol}$ Dtryptophan and 0.5 minimum inhibitory concentration (MIC) $(7.5 \mu \mathrm{g} / \mathrm{ml})$ tetracycline showed $90 \%$ reduction in biofilm formation. $5 \mathrm{mmol}$ Dmethionine shows $28 \%$ reduction and combination with tetracycline shows $41 \%$ reduction in biofilm formation of P. aeruginosa. D-leucine and Dtyrosine alone or in combination with tetracycline did not show significant anti-biofilm activity. D tryptophan-tetracycline combination could reduce $80 \%$ and $77 \%$ reduction in biofilm formation in two multi drug resistant $P$. aeruginosa clinical strains. D-tryptophan-tetracyclinecombination could also reduce $76 \%$ and $66 \%$ reduction in biofilm formation in wound dressing model and porcine skin explant respectively. The cytotoxicity and hemocompatibility studies did not show significant toxicity when this combination was used.
\end{abstract}

Conclusion: The results established the potential therapeutic application of D-tryptophan alone or in combination with tetracycline for treating biofilm associated clinical problems caused by P. aeruginosa.

Keywords: D-amino acid, Tetracycline, Biofilm, P. aeruginosa

(C) 2016 The Authors. Published by Innovare Academic Sciences Pvt Ltd. This is an open access article under the CC BY license (http://creativecommons.org/licenses/by/4. 0/) DOI: http://dx.doi.org/10.22159/ijpps.2016v8i11.9531

\section{INTRODUCTION}

Many bacteria form biofilm, which is an assemblage of surfaceassociated microbial cells enclosed in an extracellular polymeric substance matrix. Biofilm, which consists of polysaccharide, protein and DNA [1] has high significance in agricultural, industrial, environmental and clinical settings. The soil bacterium, Bacillus subtilis forms biofilm on the roots to protect plants from variety of pathogens [2]. The cause of most of the persistent infections is biofilm formation, as these groups can tolerate very high doses of antibiotics that would kill planktonic cells. $P$. aeruginosa, the most predominant pathogen in immuno-compromised patients $[3,4]$ forms biofilm virtually on any surface at any nutritional or environmental conditions. The ability to form biofilm is thought to be one of its main survival strategies when infecting a host and it is considered to be important for pathogenesis [1].

Classically biofilm development can be divided into three events, attachment, maturation and dispersion [5, 6]. The final event contributes to biological dispersal, bacterial survival, and disease transmission. It provides a mechanism whereby biofilm bacteria can spread throughout an infected organ [7] or to the whole body, in which a chronic infection can cause an acute blood stream infection [8-10]. Bacterial detachment and dispersal are triggered by various chemical signals [7]. Some of them are D-AAs, lysostaphin, farnesol, and cis-2-decenoic acid [11]. Given that many bacteria produce D-AAs, these molecules may provide a general strategy for biofilm disassembly [12]. Combining biofilm dispersal and inhibition agents with antibiotics can greatly enhance the susceptibility of bacterial biofilm to antibiotic therapy [13]. D-AAs such as Dtryptophan reported to have inhibitory effect on biofilm development by food borne organism Cronobacter sakazakii.[14]. Similarly combination of D-tyrosine with tetrakishydroxymethyl phosphonium sulphate has the ability to mitigate sulfur reducing bacterial biofilm[15]. In addition to biofilm inhibitory properties D-AAs also have other properties.
Administration of D-methionine after noise exposure leads to rescue from noise induced auditory brain stem response threshold shift [16]. Dleucine has potential anti-seizure properties. [17]. In the present study we evaluated the combinatorial effect of D-AAs with antibiotics. The antibiofilm activities of the tetracycline and D-AAs combination against P. aeruginosa strains were tested.

\section{MATERIALS AND METHODS \\ Bacterial strains and treatments}

P. aeruginosa wild strain, PA01 (P. aeruginosa ATCC 15692) obtained from ATCC and two multidrug resistant clinical strains PACL-W $(P$. aeruginosa clinical strain-surgical wound swab(leg) and PACL-S $(P$. aeruginosa clinical strain-sputum) were treated with amino acids, Dleucine, D-tryptophan, D-methionine and D-tyrosine (Sigma Aldrich USA). All the other aminoacids except D-tyrosine was dissolved in distilled water and D-tyrosine was dissolved in $1 \mathrm{~N} \mathrm{HCl}$

Antibiotic sensitivity of bacterial strains was assessed by disc diffusion method. Antibiotic discs (Himedia India) were placed on the surface of Müeller Hinton agar plates (Himedia, India). Subsequent to overnight incubation at $37^{\circ} \mathrm{C}$, growth inhibition zones formed around the disks were measured. The minimum inhibitory concentration (MIC) was evaluated by broth dilution method. Briefly, bacterial suspensions were prepared in $0.9 \%$ saline from overnight cultures and adjusted to attain the 0.5 McFarland turbidity standards. Suspensions were inoculated onto Müeller-Hinton broth containing antibiotics at concentrations 5-50 $\mu \mathrm{g} / \mathrm{ml}$ and incubated at $37^{\circ} \mathrm{C}$ for 20 to $24 \mathrm{~h} \mathrm{[18]}$.

\section{Determination of biofilm formation}

Biofilm formation was assessed by microtiter plate method [19]. Briefly, overnight bacterial cultures were diluted to 0.1 OD at 600 
nm.100 $\mu \mathrm{l}$ of diluted sample was incubated with D-AAs and $0.5 \mathrm{MIC}$ tetracycline combination in culture medium (LB broth) at $37^{\circ} \mathrm{C}$ for $24 \mathrm{~h}$ in 96-well polystyrene micro titer flat bottom plate (Nest Biotech Co. Ltd). After incubation, the medium was discarded; wells were washed with water and incubated with crystal violet $(0.1 \%)$ for $10 \mathrm{~min}$. Subsequently the plates were washed with water to remove the unbound dye. The crystal violet bound to the biofilm was eluted using 33\% acetic acid and optical density (OD) was recorded at $600 \mathrm{~nm}$ using a Synergy HT Multi-Mode Microplate Reader (BioTek Instruments, Winooski, VT). Percentage inhibition of biofilm formation was calculated using the following formula

$$
\% \text { inhibition }=\frac{\text { OD of Control }- \text { OD of test }}{\text { OD of Control }} * 100
$$

\section{Cell viability assay}

Cell viability assay was performed according to the protocol described earlier [20]. 3T3-L1 mouse fibroblast cells were cultured in Dulbecco's modified Eagle's medium (DMEM) supplemented with $10 \%$ foetal bovine serum (FBS) (v/v), 1\% penicillin, 1\% streptomycin and $0.1 \%$ amphotericin B. Cell viability assays were performed for cells treated with/without D-tryptophan in DMEM by using 3-(4,5-dimethyl-2thiazolyl)-2,5-diphenyl-2 $H$-tetrazolium bromide (MTT). 3T3-L1 cells were seeded at a density of 7500 cells/well in a 96-well microtiter plate and incubated overnight. Cells were treated with and without DTryptophan at a concentration of $5 \mathrm{mmol}$ and $10 \mathrm{mmol}$ in DMEM for 24 h. $20 \mu \mathrm{l}$ of $5 \mathrm{mg} / \mathrm{ml}$ MTT was added to each well and incubated for $3 \mathrm{~h}$ at $37{ }^{\circ} \mathrm{C}$. The media was removed after incubation and $100 \mu \mathrm{l}$ of MTT solvent ( $4 \mathrm{mmol} \mathrm{HCl}$ and $0.1 \%$ Nonidet P-40 in isopropanol) was added for solubilisation. After shaking briefly for $5 \mathrm{~min}$, the absorbance was read at $590 \mathrm{~nm}$ with a reference filter of $620 \mathrm{~nm}$ using a Synergy HT Multi-Mode Microplate Reader (BioTek Instruments, Winooski, VT).

\section{Determination of hemolysis}

Extent of hemolysis was determined according to previously described method [21]. Briefly $1 \mathrm{ml}$ blood was diluted 10 times with Ringer buffer. $200 \mu \mathrm{l}$ of diluted blood was incubated with D-tryptophan and tetracycline at $37^{\circ} \mathrm{C}$ for $24 \mathrm{~h}$ and centrifuged at 3,500 rpm for $15 \mathrm{~min}$. OD of the supernatant was recorded at $540 \mathrm{~nm}$ using microplate reader. Triton-X $100(0.01 \%)$ and $0.9 \%$ saline treated blood samples served as positive and negative controls respectively.

\section{In vitro wound dressing model}

The in vitro wound dressing model was done as previously described [22]. Overnight bacterial cultures were diluted in fresh LB broth to contain $1 \times 108$ colony forming units ( $\mathrm{CFU}) / \mathrm{ml}$ and placed on sterile polyethylene absorbent wound dressing $(1.5 \mathrm{~cm} \times 1.5 \mathrm{~cm})$ in freshly prepared LB agar plates. The plates were kept undisturbed for $30 \mathrm{~min}$ while the inoculum was allowed to dry. Fresh gauze squares $(2 \mathrm{~cm} \times 2$ $\mathrm{cm}$ ) with D-tryptophan and tetracycline were placed over the inoculated polyethylene pads and the plates were incubated at $37^{\circ} \mathrm{C}$. After $24 \mathrm{~h}$ of growth period, the samples were removed from the plates and the polyethylene pads or gauze pieces were separated and processed to determine the biofilm formation. Polyethylene pads were washed with normal saline and vortexed in saline for $1 \mathrm{~min}$. The bacterial suspension was then serially diluted and $1 \mathrm{ml}$ aliquots of each dilution were placed on LB agar plates. After $24 \mathrm{~h}$ of incubation at $37^{\circ} \mathrm{C}$, the colony forming units were calculated.

\section{Ex-vivo porcine skin model}

Ex-vivo porcine skin model was prepared according to the protocol described earlier [21, 23]. Hides from carcasses of freshly killed adult pigs obtained from a local slaughter house were thoroughly washed and de-haired. The excess fat deposits were removed and cut into sections of $2 \mathrm{~cm} \times 2 \mathrm{~cm}$. Subsequently the skin explants were washed in PBS, disinfected with $70 \%$ ethanol for $5 \mathrm{~min}$ and allowed to dry in sterile petri plates. The explants were incubated at $37^{\circ} \mathrm{C}$ for $24 \mathrm{~h}$ with $100 \mu \mathrm{l}$ bacterial suspension with/without test compounds. They were submerged in Tryptic soy broth. After incubation; sections were rinsed with sterile PBS to remove the unattached bacterial cells. Subsequently the sections were aseptically transferred to sterile test tubes containing sterile PBS with $5 \mu \mathrm{l} / \mathrm{l}$ Tween 80 and sonicated to liberate bacteria from the biofilm. This suspension was then serially diluted and $100 \mu \mathrm{l}$ aliquots of each dilution were spread on Tryptic Soy agar (TSA). After overnight incubation at $37^{\circ} \mathrm{C}$, the colonies were counted and the colony forming unit ( $\mathrm{CFU}$ ) was calculated.

\section{Statistical analysis}

Statistical analyses were performed using GraphPad Prism software. Values are expressed as mean \pm SD. Dunnett-ANOVA test was employed to compare between the test and control. Significance at ${ }^{*} p<0.05$ Vs control, ${ }^{* *} p<0.01 \mathrm{Vs}$ control, ${ }^{* * *} p<0.001$. Vs control

\section{RESULTS}

\section{Antibiotic susceptibility and minimum inhibitory concentration (MIC)}

Antibiotic susceptibility was tested using ciprofloxacin, ceftazidime, amikacin, co-trimoxazole, gentamycin, streptomycin, chloramphenicol and tetracycline. All the three P. aeruginosa strains were susceptible to tetracycline. The lowest concentration of tetracycline which inhibits the visible growth of bacterium i.e. the MIC value was found to be $15 \mu \mathrm{g} / \mathrm{ml}$. Hence sub inhibitory concentration (0.5MIC) i.e. $7.5 \mu \mathrm{g} / \mathrm{ml}$ of tetracycline was selected for further experiments. The results of MIC of tetracycline is shown in fig. 1.

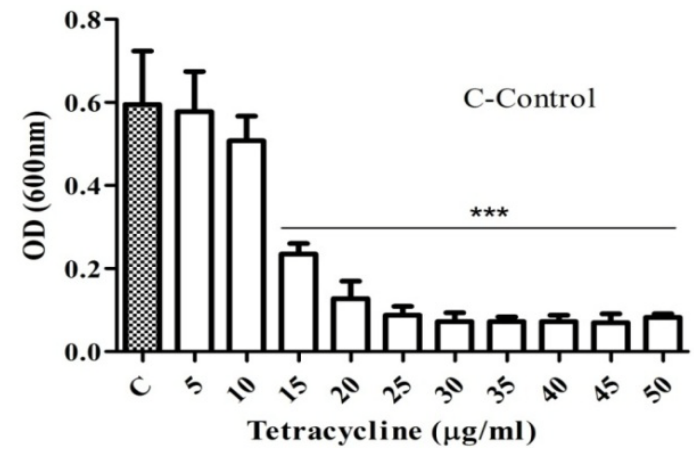

Fig. 1: Minimum inhibitory concentration (MIC) of tetracycline. MIC of tetracycline against $P$. aeruginosa was determined at a concentration range from $5-50 \mu \mathrm{g} / \mathrm{ml}$. Mean values of triplicate independent experiments and SD is shown. Dunnett's test demonstrates significant difference between the tests and the control. Significance at ${ }^{* * *} p<0.001$

Dose dependent effect of D-AAs and D-AAs-tetracycline combination on biofilm formation of $P$. aeruginosa

To evaluate the potential of D-AAs and a combination of D-AAs with tetracycline on antibiofilm activity against $P$. aeruginosa PAO1, different doses of D-AAs ranging from 5-50 mmol in combination with 0.5 MIC tetracycline $(7.5 \mu \mathrm{g} / \mathrm{ml})$ were selected for testing.(fig. 2 (A). As shown in fig. 2 (B) among the four D-AAs tested D-tryptophan showed maximum antibiofilm activity at $5 \mathrm{mmol}(61 \%)$, while D-tryptophan-tetracycline treatment showed 90\% inhibition. $5 \mathrm{mmol}$ D-methionine showed $28 \%$ inhibition and combination with tetracycline augmented the effect to $41 \%$. In contrast, D-leucine and D-tyrosine had minimal antibiofilm activity even at a concentration of $50 \mathrm{mmol}$.

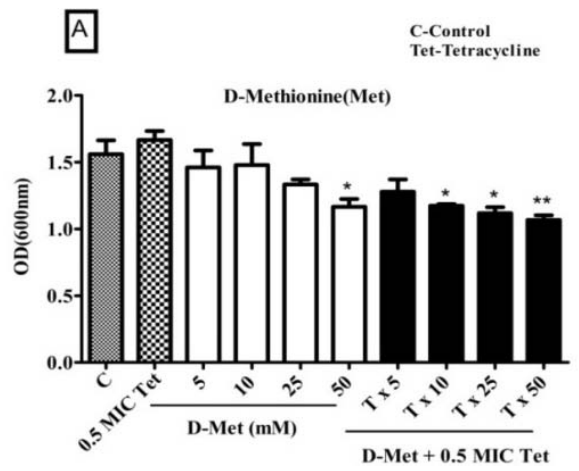


D- Tyrosine(Tyr)
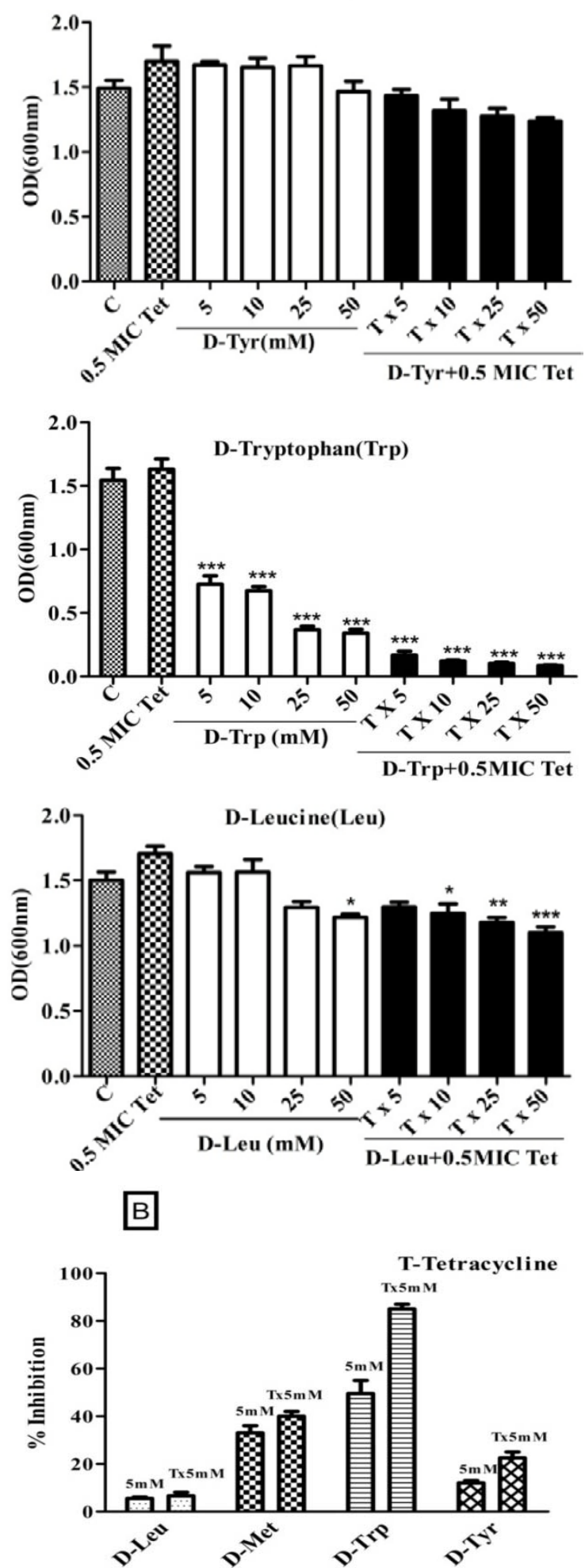

Fig. 2: (A). Dose-dependent effect of D-AAs against P. aeruginosa biofilm formation. Screening of antibiofilm activity of individual D-AAs, D-methionine, D-tyrosine, D-tryptophan and D-leucine at concentrations ranging from $5 \mathrm{mmol}$ to $50 \mathrm{mmol}$ and its combination with 0.5 MIC tetracycline $(7.5 \mu \mathrm{g} / \mathrm{ml})$ (Tet). Mean values of triplicate independent experiments and SD are shown. Dunnett's test demonstrates significant difference between the tests and the control. Significance at ${ }^{*} p<0.05,{ }^{* *} p<0.01$,

${ }^{* * *} p<0.001$, (B). Percentage inhibition of biofilm formation by $P$. aeruginosa PA01 with different D-aminoacids $(5 \mathrm{mmol})$ and its combination with $0.5 \mathrm{MIC}(7.5 \mu \mathrm{g} / \mathrm{ml})$ tetracycline (T)
D-tryptophan-tetracycline combination disperses the biofilm formed by clinical isolates of $P$. aeruginosa

Treatment with 5 mmol D-tryptophan-0.5 MIC tetracycline combination on multi-drug resistant $P$. aeruginosa strains PACL-S $(P$. aeruginosa clinical strain-sputum) and PACL-W ( $P$. aeruginosa clinical strain-leg wound swab) demonstrated significant anti biofilm activity. This activity of D-AAs against biofilm was partly strain dependent. Treatment with $5 \mathrm{mmol}$ tryptophan inhibited biofilm formation in PACLW and PACLS by $51 \%$ and $50.5 \%$ respectively, while treatment with D-tryptophan and tetracycline in combination augmented this effect by $80 \%$ and $77 \%$ respectively in PACLW and PACLS (fig. 3).

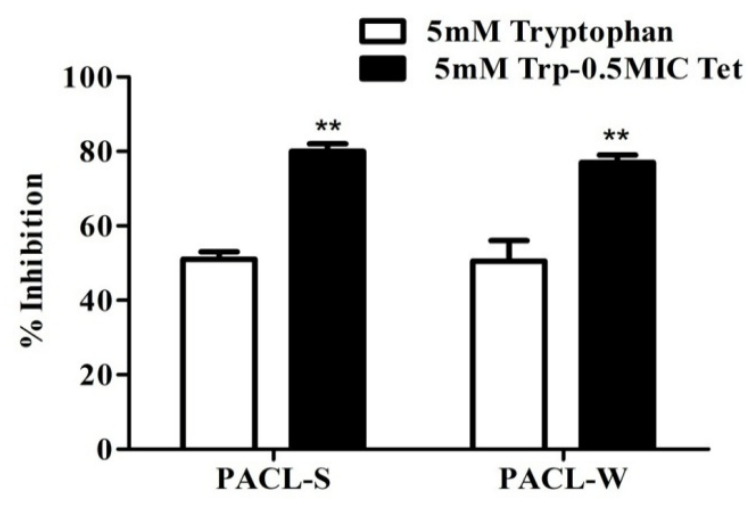

Fig. 3: Percentage inhibition of the biofilm formed by clinical strains of $P$. aeruginosa. $P$. aeruginosa strains isolated from sputum (PACL-S) and wound (PACL-W) treated with 5 mmol

tryptophan (Trp) alone or in combination with 0.5 MIC

tetracycline (Tet). Mean values of triplicate independent experiments and SD are shown

In vitro wound dressing model and ex-vivo porcine skin model

To better understand anti-biofilm activity of D-tryptophantetracycline combination in other biofilm models, we used in vitro wound dressing and ex-vivo porcine skin models. In wound dressing model, $5 \mathrm{mmol}$ D-tryptophan in combination with 0.5 MIC tetracycline reduced biofilm formation (76\%) when compared to controls (fig. 4).

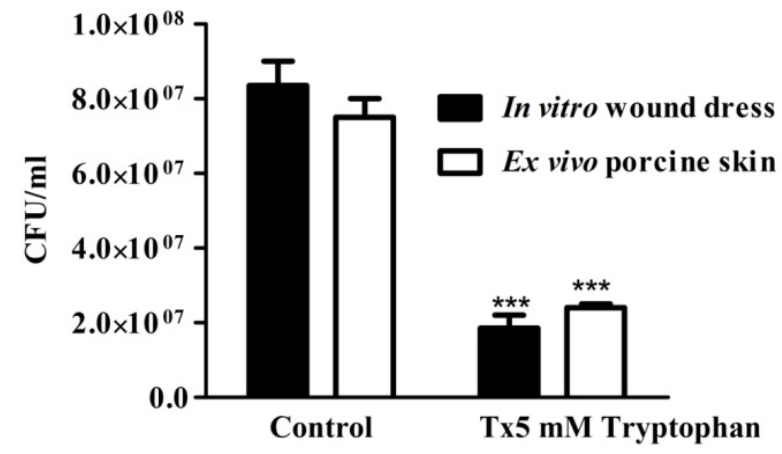

Fig. 4: Effect of D-tryptophan and 0.5 MIC tetracycline combination on the biofilm formed by $P$. aeruginosa using in vitro wound dress model and ex vivo porcine skin model. Wound dress/porcine skin inoculated with bacterial cultures were treated with $5 \mathrm{mmol}$ tryptophan and 0.5 MIC tetracycline. Planktonic bacteria were removed and the bacteria present in the biofilm were detected by plated into LB agar plates. Mean values of triplicate independent experiments and SD are shown

Further, the anti-biofilm activity of 5 mmol D-tryptophan in combination with 0.5 MIC tetracycline was tested using ex-vivo porcine skin model. Consistent with the previous result, D- 
tryptophan in combination with tetracycline inhibited biofilm formation in porcine skin explants by $66 \%$ compared to the untreated explants (fig. 4). These results confirm the efficacy of Dtryptophan-tetracycline combination in inhibiting biofilm formation.

\section{Cell viability assay}

To investigate the effect of D-tryptophan on viability of cells, MTT assay was performed.3T3-L1 fibroblast cells were incubated with 5 $\mathrm{mmol}$ and $10 \mathrm{mmol}$ D-tryptophan with/without $0.5 \mathrm{MIC}$ tetracycline for $24 \mathrm{~h}$. Results from MTT analysis showed no significant cytotoxicity on treatment when compared to the untreated control (fig. 5).

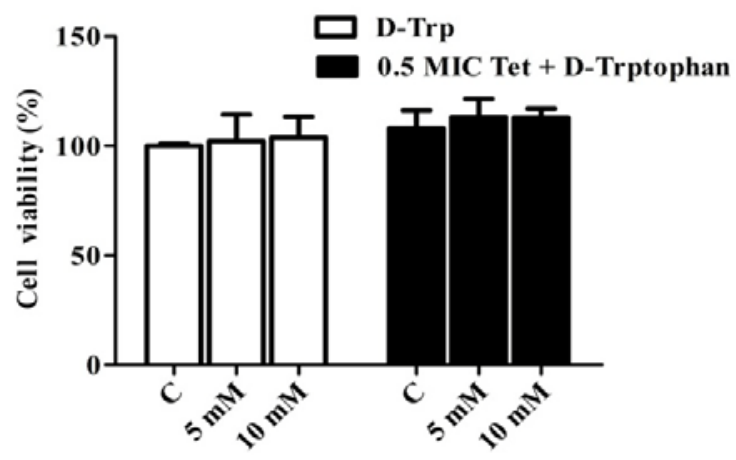

Fig. 5: Effect of D-tryptophan in combination with 0.5 MIC tetracycline on cell viability of 3T3-L1 fibroblast cells. Cell viability using MTT cytotoxicity assay was performed for the 3T3-L1 cells treated with D-tryptophan in combination with 0.5 MIC tetracycline in DMEM supplemented with $10 \%$ FBS. Mean values of triplicate independent experiments and SD are shown. Dunnett's test demonstrates no significant difference between the test and the control

\section{Hemocompatibility testing of D-tryptophan in combination with tetracycline}

The hemocompatibility of $5 \mathrm{mmol}$ D-tryptophan-0.5 MIC tetracycline mixture was analyzed. No significant hemolysis was observed when this combination was used (fig. 6).

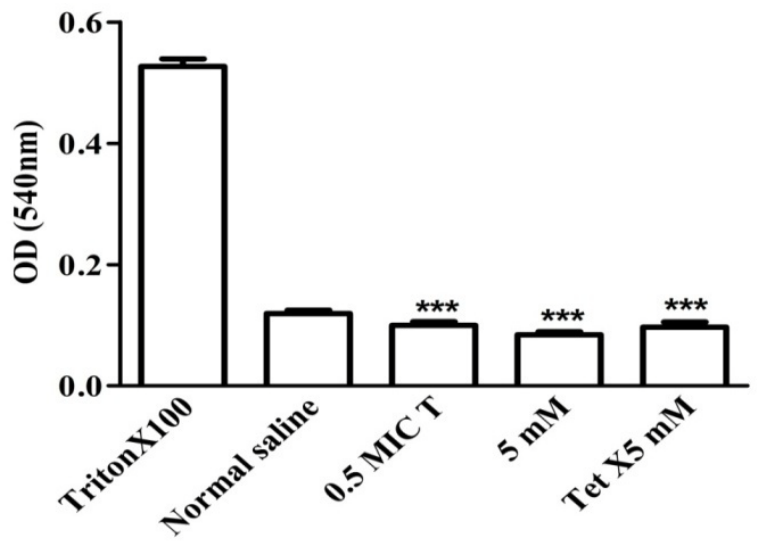

Fig. 6: Hemocompatibility of D-Tryptophan in combination with 0.5 MIC tetracycline (Tet). A representative bar diagram showing hemolysis by Triton $X 100$, normal saline, 0.5 MIC tetracycline, 5 mmol tryptophan and 5 mmol tryptophan-0.5 MIC tetracycline combination. Mean values of triplicate independent experiments and SD are shown. Dunnett's test establishes significant difference between the test and the control. Significance at ${ }^{* * *} p<0.001$ Vs control

\section{DISCUSSION}

Most of the commercially available antibiotics are developed against planktonic and metabolically active bacteria. However, bacteria in the biofilm are different and can be 10-1,000 times more resistant to antibiotics than planktonic bacteria [24]. Designing an anti-biofilm strategy is complicated due to the characteristic properties possessed by biofilm [25] that include protective shielding by the matrix, specific expression of microbial proteins for nutrient scavenging, ramping down of metabolic activity, induction of a secluded state in which bacterial cells are in a phenotypically variable state and resistant to antimicrobial treatment [26,27].

Recent studies have pointed out the possible use of antibiofilm/biofilm dispersing agents like D-AAs as potential therapeutic molecules [12] Combining these agents with antibiotics can have significant promising effects. In Bacillus subtilis, a mixture of D-AAs (D-leucine, Dmethionine, D-tyrosine and D-tryptophan) is reported to prevent biofilm formation and induce existing biofilm disassembly [11] Previous studies have reported anti-biofilm activities of D-AAs in Staphylococcus aureus [12] and P. aeruginosa [13]. These results indicate that D-AAs might prove widely useful in medical and industrial applications for the prevention or eradication of biofilm.

Consistent with previous studies [13], D-tryptophan showed highest antibiofilm activity followed by D-methionine, while marginal activity was observed in the case of D-leucine and D-tyrosine. A dose dependent antibiofilm activity was seen in the presence of Dtryptophan. Although tetracycline alone did not show any significant activity against biofilm at 0.5 MIC, combination of tetracycline and D-AAs showed enhanced antibiofilm activity. We also observed that co-treatment of D-tryptophan-tetracycline had promising antibiofilm effect compared to other combinations. Our results are in line with previous studies that have demonstrated that the activity of various antimicrobial agents can be significantly enhanced by the addition of dispersal agents [28].

As a model designed to assess the efficacy of topical application of tetracycline and D-tryptophan combination, gauze squares saturated with this combination was checked for their antibiofilm activity. The decreased bacterial load recovered from the dressing containing 5 mmol D-tryptophan-0.5 MIC tetracycline mixture when compared to the untreated control supported the observations from the biofilm tests done in microtitre plate. Further, the antibiofilm activity of Dtryptophan-tetracycline combination was validated using porcine skin infection model. Porcine skin model for bacterial biofilm attachment was selected because it fulfills the requirements of an $e x$ vivo model due to its similarity with human skin physiology. The other advantage of using such a model is that a large number of trials can be conducted economically [23]. Our study clearly elucidated that a single application of $5 \mathrm{mmol}$ D-tryptophan-0.5 MIC tetracycline combination was capable of reducing $P$. aeruginosa biofilm attachment to porcine skin explants. Cell viability assay performed in 3T3-L1 fibroblast cells showed that $5 \mathrm{mmol}$ and 10 mmol D tryptophan in combination with 0.5 MIC tetracycline did not have any cytotoxic effect. The results of hemocompatibility studies were also in strong agreement with the cytotoxicity studies suggesting the potential of D-tryptophan-tetracycline combination as an effective therapy against $P$. aeruginosa infections.

The mechanism through which the D-AAs and its combination with antimicrobials act against biofilm have not been fully addressed yet. It is possible that D-AAs cause altered protein expression, disrupt cell wall integrity and metabolic status. A recent study reported that epigallocatechin gallate,the major catechin present in green tea extract enhances tetracycline activity against resistant staphylococcal isolates by impairment of tetracycline efflux pump activity thereby increasing intracellular retention of the drug leading to effective synergistic drug combination $[29,30]$. The results obtained demonstrate that antibiotic treatment with D-AAs could be more effective to reduce infections with minimal systemic toxicity. Moreover antibiotic concentrations required could be reduced by co-delivery with D-AAs, by which antibiotic toxicity can potentially reduced.

\section{CONCLUSION}

In conclusion, we have demonstrated in vitro anti-biofilm activity by D-AAs in P. aeruginosa. Anti-biofilm activity by individual amino 
acids was dose dependent, and amino acids in combination with tetracycline potentiated the anti-biofilm activity against diverse isolates of $P$. aeruginosa. The in vitro studies were validated using $e x$ vivo porcine skin model. This combination may find commercial advantages in topical applications and treatment of infections caused by $P$. aeruginosa.

\section{ACKNOWLEGMENT}

The authors graciously acknowledge the chancellor Sri Mata Amritanandamayi for the constant motivation. The authors would like to thank the BRITE initiative at Amrita School of Biotechnology for the financial support.

\section{CONFLICT OF INTERESTS}

\section{Declared none}

\section{REFERENCES}

1. Høiby N, Bjarnsholt T, Givntskov M, Ciofu O. Antibiotic resistance of bacterial biofilms. Int J Antimicrob Agents 2010;35:322-32.

2. Nagorska K, Bikowski M, Obuchowski M. Multicellular behavior and production of a wide variety of toxic substances support usage of Bacillus subtilis as a powerful bio control agent. Acta Biochim Pol 2007;54:495-508.

3. Bjarnsholt T, Ciofu O, Molin S, Givskov M, Hoiby N. Applying insights from biofilm biology to drug development-can a new approach be developed? Nat Rev Drug Discovery 2013;12:791808.

4. Van Delden C, Iglewski H. Cell-to-cell signaling and Pseudomonas aeruginosa infections. Emerging Infect Dis 1998;4:551-60.

5. Sauer K, Cullen MC, Richard AH, Zeef LA, Davies DG, Gilbert P. Characterization of nutrient induced dispersion in $P$. aeruginosa PA01 biofilm. J Bacteriol 2004;186:7312-26.

6. Karatan E, Watnick P. Signal regulatory networks andmaterials that build andbreak bacterial biofilm. Microbiol Mol Biol Rev 2009;73:310-47.

7. Costerton JW, Stewart PS, Greenberg EP. Bacterial biofilm: a common cause of persistent infections. Science 1999;284:1318-22.

8. Rupp CJ, Fux CA, Stoodley P. Viscoelasticity of Staphylococcus aureus biofilm in response to fluid shear allows resistance to detachment and facilitates rolling migration. Appl Environ Microbiol 2005;71:2175-8.

9. Abraham J, Mansour C, Veledar E, Khan B, Lerakis S. Staphylococcus aureus bacteremia and endocarditis: the Grady Memorial Hospital experience with methicillin-sensitive $S$ aureus and methicillin-resistant $S$ aureus bacteremia. Am Heart J 2004;147:536-9.

10. Fatkenheuer G, Cornely O, Seifert H. Clinical management of catheter-related infections. Clin Microbiol Infect 2002;8:545-50.

11. Kolodkin-Gal I, Romero D, Cao S, Clardy J, Kolter R, Losick R. Daminoacids trigger biofilm disassembly. Science 2010;328:627-29.

12. Hochbaum IA, Kolodkin-Gal I, Folston L, Kolter R, Aizen, Losick R. Inhibitory effect of D-amino acids on $S$. aureus biofilm development. J Bacteriol 2011;193:5616-22.

13. Brandenberg SK, Rodriguez JK, Mc Anulty JF, Murphy JC, Abbott LN, Schurr JM, et al. Tryptophan inhibits biofilm formation by $P$. aeruginosa. Antimicrob Agents Chemother 2013;57:1921-2.

14. Li H, Ye Y, Ling N, Wu Q, Zhang J. Inhibitory effect of Dtryptophan on biofilm development by the food borne pathogen Cronobactersakazakii. Int Dairy J 2015;49:125-9.
15. Xu D, Li Y, Gu TA. Synergistic D-tyrosine and tetrakishydroxy methyl phosphoniumsulphate biocide combination for the mitigation of SRB biofilm. World J Microbiol Biotechnol 2012;28:3067-74.

16. Campbell K, Claussen A, Meech R, Verhulst S, Fox D, Hughes L. D-methionine (D-met) significantly rescues noise-induced hearing loss: timing studies. Hear Res 2011;282:138-44.

17. Hartman AL, Santos P, O' Riordan KJ, Stafstorm CE, Marie Hardwick J. Potent anti-seizure effect of D-leucine. Neurobiol Dis 2015;82:46-53.

18. Andrew MJ. Determination of minimum inhibitory concentration. J Antimicrob Chemother 2001;48:5-15.

19. AdonizioAL, Kong KF, Mathee K. Inhibition of quorum sensing controlled virulence factor production in P. aeruginosa by South Florida plant extract. Antimicrob Agents Chemother 2008;52:198-203.

20. Omanakuttan A, Nambiar J, Harris MR, Bose C, Pandurangan N, Varghese RK, et al. Anacardic acid inhibits the catalytic activity of matrix metalloproteinases-2 and metalloproteinases 9. Mol Pharmacol 2012;82:614-22.

21. Nataraj N, Anjusree GS, Madhavan AA, Priyanka P, Sankar D, Nisha $\mathrm{N}$, et al. Synthesis and anti-staphylococcal activity of TiO2 nanoparticles and nanowires in Ex Vivo porcine skin model. J Biomed Nanotechnol 2013;9:1-7.

22. Hammond AA, Miller KG, Kruczek CJ, Dertien J, Colmer Hamood JA, Griswold JA, et al. An in vitro biofilm model to examine the effect of antibiotic ointments on biofilm produced by burn wound bacterial isolates. Burns 2011;37:312-21.

23. Yang Q, Phillips PL, Sampson EM, Progulske-Fox A, Jin $\mathrm{S}$, Antonelli P, et al. Development of a novel ex vivo porcine skin explant model for the assessment of mature bacterial biofilm. Wound Repair Regeneration 2013;21:704-14.

24. Davey ME, O'Toole GA. Microbial biofilm: from ecology to molecular genetics. Microbiol Mol Biol Rev 2000;64:847-67.

25. Donlan RM, Costerton JW. Biofilm: survival mechanisms of clinically relevant microorganisms. Clin Microbiol Rev 2002;15:167-93.

26. Davies D. Understanding biofilm resistance to antibacterial agents. Nat Rev Drug Discovery 2003;2:114-22.

27. Fux CA, Costerton JW, Stewart PS, Stoodley P. Survival strategies of infectious biofilms. Trends Microbiol 2005;13:3440.

28. Sanchez CJ Jr, Akers KS, Romano DR, Woodbury RL, Hardy SK, Murray CM, et al. D-amino acids enhance the activity of antimicrobials against biofilm of clinical wound isolates of Staphylococcus aureus and Pseudomonas aeruginosa. Antimicrob Agents Chemother 2014;58:4353-61.

29. Sudano A, Roccaro AS, Blanco AR, Giulian F, Rusciano D, Enea V. Epigallocatechin-gallate enhances the activity of tetracycline in Staphylococci by inhibiting its efflux from bacterial cells. Antimicrob Agents Chemother 2004;48:1968-73.

30. Jayaraman P, Sakharkar KM, Lim CS, Tang TH, Kishore R, Sakharkar RK. Activity and interactions of antibiotic and phytochemical combinations against Pseudomonas aeruginosa in vitro. Int J Biol Sci 2010;6:556-68.

\section{How to cite this article}

- $\quad$ H Jayalekshmi, C Harikrishnan, Sajin Sali, N Kaushik, Norin Mary G Victus, R Anoop, TM Sarath, O Athira, Geetha B Kumar, Bipin Nair. Combinatorial effect of d-aminoacids and tetracycline against Pseudomonas aeruginosa biofilm. Int J Pharm Pharm Sci 2016;8(11):216-220. 